\title{
The Effect of Chitosan on the In Vitro Biological Performance of Chitosan-Poly(butylene succinate) Blends
}

\author{
Daniela F. Coutinho, ${ }^{*}$ Iva H. Pashkuleva, Catarina M. Alves, Alexandra P. Marques, \\ Nuno M. Neves, and Rui L. Reis \\ 3B's Research Group-Biomaterials, Biodegradables and Biomimetics, Department of Polymer \\ Engineering, University of Minho, Campus of Gualtar, 4710-057 Braga, Portugal, IBB-Institute for \\ Biotechnology and Bioengineering, PT Government Associated Laboratory, Braga, Portugal
}

Received September 1, 2007; Revised Manuscript Received January 7, 2008

\begin{abstract}
Chitosan blends with synthetic biodegradable polymers have been proposed for various biomedical applications due to their versatile mechanical properties and easier processing. However, details regarding the main surface characteristics that may benefit from the blending of these two types of materials are still missing. Hence, this work aims at investigating the surface properties of chitosan-based blends, illustrating the way these properties determine the material-proteins interactions and ultimately the behavior of osteoblast-like cells. The surface characteristics of modified and nonmodified blends were assessed using complimentary techniques such as optical microscopy, scanning electron microscopy (SEM), Fourier transform infrared spectroscopy (FTIR-ATR), X-ray photoelectron spectroscopy (XPS), contact angle measurements and surface energy calculations. The adsorption of human serum albumin (HSA) and human plasma fibronectin (HFN) onto the different surfaces was quantified by association of an indirect method with a colorimetric assay. It was found that the presence of chitosan on the surface promoted the adsorption of proteins. Moreover, a preferential adsorption of albumin over fibronectin was registered. The in vitro biological performance of the studied materials was further investigated by a direct contact assay with an osteoblastic-like cell line (SaOs-2). A synergistic effect of the two components of the blend was observed. While the synthetic polyester promoted the adhesion of SaOs-2, the presence of chitosan significantly enhanced the osteoblastic activity of these cells. This work further confirmed the interest in designing polymeric blends with natural polymers as a successful strategy to enhance the biological performance of a biomaterial.
\end{abstract}

\section{Introduction}

Natural polymers received great attention in the biomaterials field. Their structural similarity with biological macromolecules makes them easily recognized by the bioenvironment and therefore easily metabolized to residues that are noncytotoxic and naturally eliminated. Chitosan is one of the natural polymers that have been widely studied for biomedical applications. ${ }^{1}$ Some of the main advantages of chitosan are its biodegradability, antimicrobial, and antitumor activity ${ }^{2,3}$ as well as its relative low cost because it is obtained by alkaline $N$-deacetylation of chitin, the second most abundant polysaccharide after cellulose. ${ }^{2}$ However, chitosan also presents some drawbacks: it is difficult to process ${ }^{4}$ and generally shows limitations in supporting cell adhesion and proliferation. ${ }^{5,6}$ Many strategies have been proposed to overcome these limitations. ${ }^{2,3,7} \mathrm{~A}$ widely used strategy is the blending of chitosan with other polymers possessing complementary characteristics such as processability, functionality, degradability, and mechanical properties that can not be achieved by the individual polymer. Melt blends of chitosan with the biodegradable aliphatic polyesters have been already studied $^{8,9}$ in terms of their bulk properties and shown to have a great potential to be used in the biomaterials field. However, the biological response to any implanted biomaterial is highly dependent on its surface properties. ${ }^{10,11}$ The surface of the biomaterial is the first to become in contact with the biological fluids, hence determining the material cross-talk with proteins,

* Corresponding author. E-mail: d.coutinho@ dep.uminho.pt. Telephone: +351 253 604783. Fax: +351 253 604492. Address: 3B's Research Group-Biomaterials, Biodegradables and Biomimetics, Campus of Gualtar, 4710-057 Braga, Portugal. cells, and surrounding host tissues. During this contact, protein adsorption occurs almost instantaneously and the layer of adsorbed proteins further mediates and controls the biological performance of the material. Thus, protein adsorption has a fundamental role in dictating the cellular response elicited by biomedical systems implanted in the human body. The ability to first understand and further control those events at the biomaterial surface largely determines the biological performance of biomedical systems.

This study reports on the surface properties of a blend of chitosan with poly(butylene succinate) complemented by the analysis of the interaction of the surface both with model proteins and cells. Surface properties such as roughness, ${ }^{12,13}$ wettability, or chemistry, ${ }^{6}$ which are known to control both protein adsorption and cell response to a biomaterial, were evaluated. Etchings by polishing or by plasma were both performed in order to evaluate the difference between the surface and the bulk of the samples. The adsorption of human serum albumin (HSA) and human plasma fibronectin (HFN) onto the different surfaces was quantified by coupling an indirect method with a colorimetric assay. The in vitro biological performance of the studied materials was further investigated by means of a direct contact assay with osteoblastic-like cells (SaOs-2).

\section{Materials and Methods}

2.1. Studied Materials. The materials used in this study were a commercially available poly(butylene succinate) (PBS, Bionolle 1050, Showa High Polymer Co. Ltd., Tokyo, Japan) and its blend with chitosan (50/50 wt \%, CHT/PBS) (Figure 1). Chitosan with a degree of deacetylation of about $85 \%$ was purchased from France-chitin 

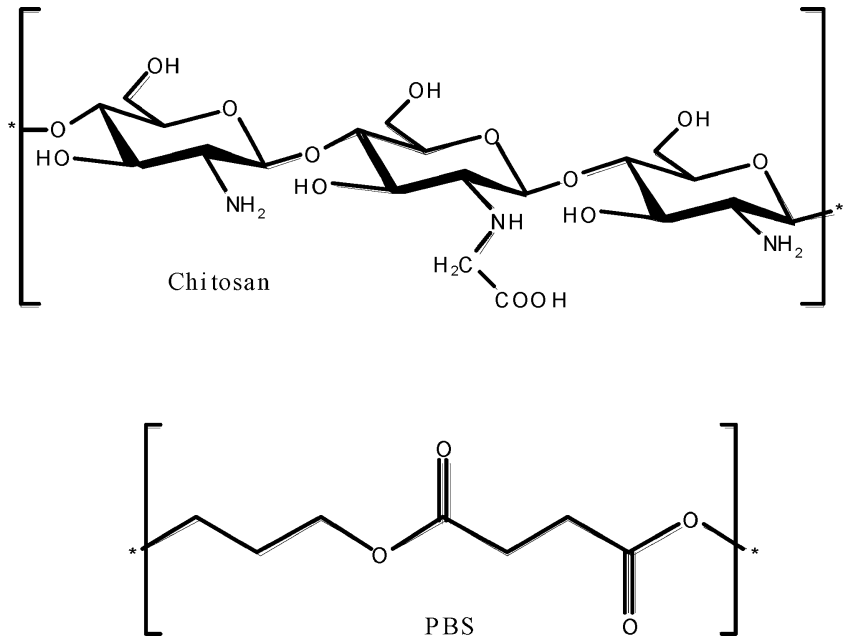

Figure 1. Chemical structures of the components of the studied blend CHT/PBS.

(Orange, France). Samples of PBS and CHT/PBS (50/50\%) were processed into circular disks $(\varnothing=10 \mathrm{~mm}$, height $=1.5 \mathrm{~mm})$ by conventional injection molding technology using optimized processing conditions. Further details on the production and the mechanical characteristics of the studied materials can be found elsewhere. ${ }^{8}$

2.2. Surface Etching. Two types of surface treatments were performed: mechanical etching by polishing using a Struers RotoPol21 machine and plasma etching in Ar atmosphere (30 W, 15 min, 0.18 mbar, PlasmaPrep5, Germany). Plasma etching was applied in order to remove a very thin (angstrom level) layer from the material surface. In turn, mechanical etching reaches a deeper region of the sample therefore allowing to evaluate the differences between the bulk and the surface properties. Changes in the surface chemistry introduced by the plasma etching were evaluated and compared with those induced by the polishing treatment.

2.3. Surface Characterization. Chitosan distribution in the polymer matrix was assessed by eosin staining. ${ }^{14}$ Discs of the blend and of PBS (control) were stained with an Eosin Y (Sigma, USA) solution for $3 \mathrm{~h}$ at room temperature. The stained samples were further examined by light microscopy (Olympus BH-2) in reflection mode and images were obtained using a microscope digital camera Olympus DP11. The surface morphology of the samples was analyzed using a Leica Cambridge S-360 scanning electron microscope (SEM, Leica Cambridge, UK). All specimens were precoated with a conductive layer of sputtered gold. The micrographs were taken at an accelerating voltage of $15 \mathrm{kV}$ at different magnifications.

The chemistry of the surfaces was analyzed by Fourier transform infrared spectroscopy with attenuated total reflection (FTIR-ATR). The infrared spectra were recorded on a spectrophotometer IR-Prestige-21 (Shimadzu, Japan) with a resolution of $4 \mathrm{~cm}^{-1}$. The results are presented as the average of 32 scans. X-ray photoelectron spectroscopy (XPS) was performed for a detailed analysis of the surface chemical composition of the modified and nonmodified samples. The XPS analysis was performed using an ESCALAB 200A, VG Scientific (UK) with PISCES software for data acquisition and analysis. An achromatic $\mathrm{Al}(\mathrm{Ka}) \mathrm{X}$-ray source operating at $15 \mathrm{kV}(300 \mathrm{~W})$ was used, and the spectrometer, calibrated with reference to $\mathrm{Ag} 3 \mathrm{~d} 5 / 2(368.27 \mathrm{eV})$, was operated in CAE mode with $15 \mathrm{eV}$ pass energy. The data acquisition was performed with a pressure lower than $1.0 \times 10^{-6} \mathrm{~Pa}$. XPS analyses were performed at a takeoff angle of $90^{\circ}$ (normal to the surface). The wettability of the surfaces was assessed by contact angle measurements. Measurements of dynamic contact angle were carried out by a goniometer OCA15+ (DataPhysics, Germany) using the sessile drop (needle in) method. The used liquids $\left(\mathrm{H}_{2} \mathrm{O}\right.$ and $\mathrm{CH}_{2} \mathrm{I}_{2}, 3 \mu \mathrm{L}$, HPLC grade) were added by a motor driven syringe at room temperature. Three specimens per sample were used and three measurements were performed in each sample. The surface free energy $(\gamma)$ of treated and untreated samples was calculated by the Owens, Wendt, Rabel, and Kaelble (OWRK) method. ${ }^{15,16}$ Tissue culture polystyrene (TCPS) was used as a standard because it is the most widely used surface for cell culture studies.

2.4. Protein Adsorption Study. Two different proteins, human plasma fibronectin (HFN, Sigma, USA) and human serum albumin (HSA, Sigma, USA) were used in the present study. While HFN was used as a model adhesive protein, the HSA was chosen because of its nonadhesive properties and high concentration in physiological fluids. The affinity of these two model proteins to the studied surfaces was evaluated by their adsorption from the solution of the respective protein, using a concentration of $0.8 \mu \mathrm{g} / \mathrm{mL}$. Alternatively, a study using $0.2 \%$ $(\mathrm{v} / \mathrm{v})$ of the concentration of these proteins in the blood plasma (70 $\mu \mathrm{g} / \mathrm{mL}$ of HSA and $0.8 \mu \mathrm{g} / \mathrm{mL}$ of HFN) was conducted in order to simulate the in vivo process. ${ }^{17}$

Materials were incubated in $1 \mathrm{~mL}$ of each protein solution for $2 \mathrm{~h}$ at $37{ }^{\circ} \mathrm{C}$. TCPS surface was used as a control surface and phosphate buffered saline solution (PBS, Sigma, USA) was used as a blank solution. The adsorption of HSA and HFN onto the different surfaces was indirectly quantified by associating the depletion method with a colorimetric assay. Briefly, the amount of protein in the supernatant was quantified using the Micro-Bicinchronic Acid kit (BCA, Pierce, USA) and reading the visible absorbance $(562 \mathrm{~nm})$ in a microplate ELISA reader (BioTek, USA). The amount obtained for the supernatant was subtracted from the initial amount of the protein in the solution.

\subsection{Attachment and Proliferation of Osteoblast-Like Cells.}

2.5.1. Cell Culture. The human osteosarcoma cell line SaOs-2, with osteoblastic phenotype, was obtained from the European Collection of Cell Cultures (ECCC, UK). The cells were cultured in Dulbecco's Modified Eagle's medium (DMEM, Sigma, USA) with phenol red supplemented with $10 \%$ of heat-inactivated fetal bovine serum (FBS, Biochrom AG, Germany) and $1 \%$ of antibiotic (Gibco, USA) at $37{ }^{\circ} \mathrm{C}$ in a humidified atmosphere with $5 \% \mathrm{CO}_{2}$.

A cell suspension of SaOs-2 $\left(3.3 \times 10^{4}\right.$ cells $\left./ \mathrm{mL}\right)$ was prepared by trypsinisation ( $0.25 \%$ trypsin/EDTA solution, Sigma, USA). Three samples per material were placed in 24-well plates and $1.5 \mathrm{~mL}$ of the cell suspension was seeded onto each well. The cells, seeded at the surface of the materials, were cultured for different time periods $(1,3$, and 7 days) in order to follow the behavior of the cells on the various surfaces under study. Culture medium was changed every 3 days and TCPS was used as a control.

2.5.2. Cell Morphology Analysis. After each incubation period, the samples were washed with PBS solution, fixed with $2.5 \%$ glutaraldehyde (Sigma, USA) and kept at $4{ }^{\circ} \mathrm{C}$ in PBS until being stained or prepared for SEM observation. Methylene blue was used to stain the viable cells (Axioplan 2 microscope, Zeiss, Germany). SEM analysis was performed to obtain further details about cell morphology after the different time periods. Prior to the analysis, samples were dehydrated using graded ethanol solutions $(50 \%, 70 \%, 90 \%$, and 100\%) and hexamethyldisilazane (HMDS, Electron Microscopy Sciences, USA).

2.5.3. DNA and Alkaline Phosphatase (ALP) Activity Quantification. Cells were lysed by osmotic and thermal shock and the obtained supernatant was used for both DNA and ALP analyses.

Cell proliferation was evaluated by quantifying the DNA content along the time of culture using the PicoGreen dsDNA kit (Molecular Probes) and according to the instructions of the manufacturer. Fluorescence was read $(485 \mathrm{~nm} / 528 \mathrm{~nm}$ of excitation/emission) in a microplate ELISA reader (BioTek, USA), and the DNA amounts calculated from a standard curve.

The effect of the surface over ALP activity, a marker of osteoblastic activity, was evaluated using the $p$-nitrophenol assay. ${ }^{18}$ The optical density was determined at $405 \mathrm{~nm}$ in a microplate ELISA reader (BioTek, USA) and the activity of the enzyme extrapolated from a standard curve.

2.6. Statistical Analysis. The quantification of adsorbed proteins, DNA quantification and ALP activity were performed in three separate sets of experiments with three replicates for each material. All the data 

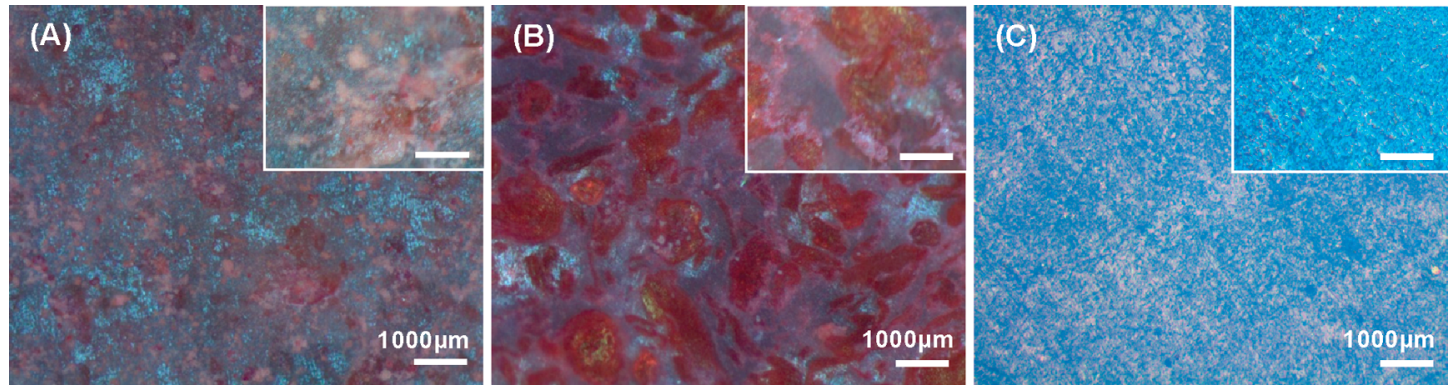

Figure 2. Optical micrographs of $C H T / P B S$ surface (A), CHT/PBS bulk (after polishing) (B), and PBS (C) after staining with Eosin $Y$ dye. Bar for the higher magnification micrographs corresponds to $500 \mu \mathrm{m}$.
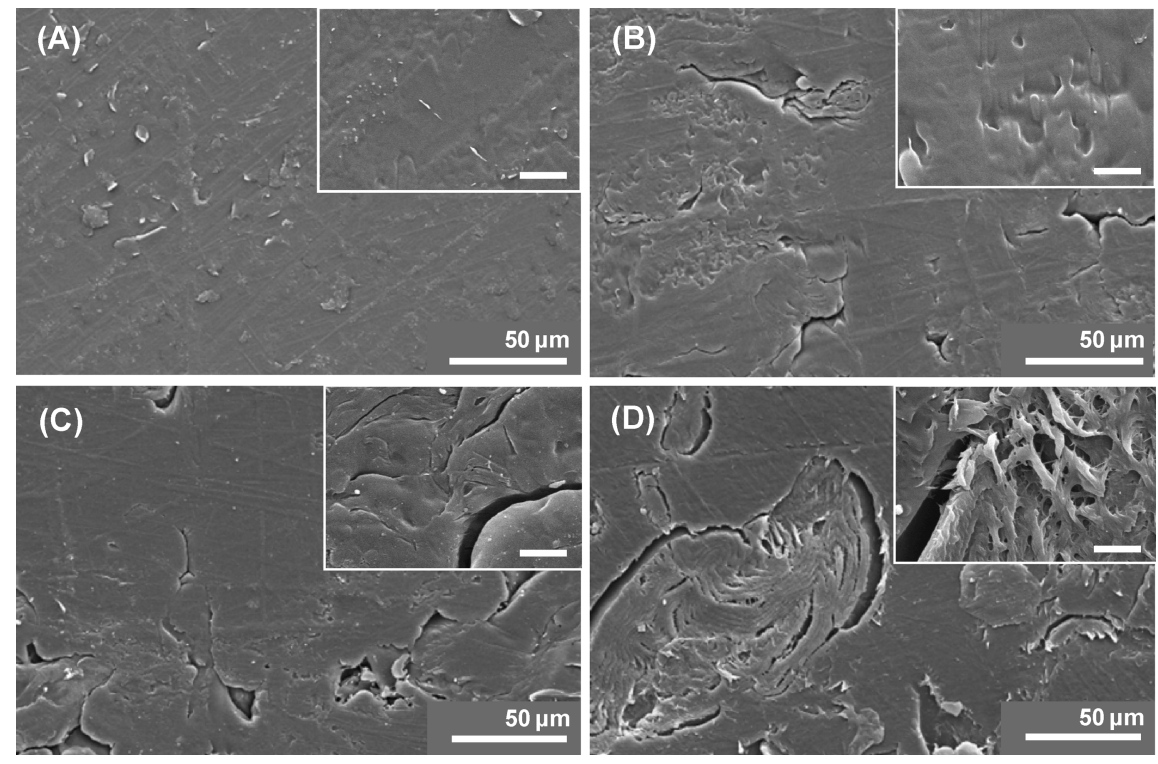

Figure 3. Scanning electron micrographs of PBS (A), CHT/PBS (B), plasma etched CHT/PBS (C), and polished CHT/PBS (D). Bar for the higher magnification micrographs corresponds to $5 \mu \mathrm{m}$.

were subjected to statistical analysis and were reported as mean \pm standard deviation. The normality of the data was checked by applying the Shapiro-Wilk's $W$-test. For the samples following a normal distribution, Student $t$-tests for independent samples were performed. The differences were considered statistically significant if $p<0.05$.

\section{Results and Discussion}

The surface composition and the surface properties of a blended material depend on the blend composition, degree of the miscibility of the system and also on the used processing conditions. Generally, for injection molding processed materials a skin-core morphology is observed. The outside layer, the so-called skin, can vary in thickness and in composition because of the polymer melt flow inside the mold.

In fact, a morphology with synthetic component rich skin was already observed ${ }^{8}$ for melt processed chitosan and aliphatic polyester blends. Because chitosan does not melt during the processing, its viscosity does not significantly change with temperature and during flow, thus causing the aggregation of the chitosan in the center of the melt flowing channels. In opposition, the synthetic component of the blend that melts during processing constitutes the continuous phase of the blend. In this case, the hydrodynamic of flow tended to produce a film at the surface which was rich in the melting component. Figure 2 represents the chitosan distribution within the studied chitosan/ PBS blend. As it was expected, this blend also presented a skin-core morphology. While the skin was rich in the PBS component (Figure 2A), chitosan nodules dispersed in the PBS continuous phase were observed in deeper layers after the polishing of the sample (Figure 2B).

Those results were further confirmed by SEM analysis (Figure 3). A planar surface with minor irregularities was observed for the PBS samples (Figure 3A). The incorporation of chitosan resulted in a more irregular and rough surface corresponding to the presence of chitosan particles underneath the surface (Figure 3B).

Plasma treatment affected the surface morphology at the nano scale (AFM study, results not shown). However, at the microscale, no significant changes in the surface morphology were observed (Figure 3C). Chitosan particles dispersed in the continuous PBS phase with the characteristic laminar morphology were observed after polishing (Figure 3D).

To further investigate the difference between the surface and the bulk of the material, FTIR analysis was performed (Figure 4, Table 1). The characteristic absorption bands of the studied blend and its components are summarized in Table 1.

As can be seen in Figure 4, FTIR-ATR spectra did not show any noticeable difference between the bulk and the surface of the CHT/PBS blend. Moreover, the spectrum of the synthetic component coincided with the one of the blended material, in which the lower intensity chitosan bands were overlapped by the much sharper and higher intensity PBS bands.

Because the FTIR-ATR spectroscopy was not sufficiently detailed to allow comparing the variation in chemical composition between the surface and the bulk, X-ray photoelectron spectroscopy (XPS), which enables analysis of outer region with 


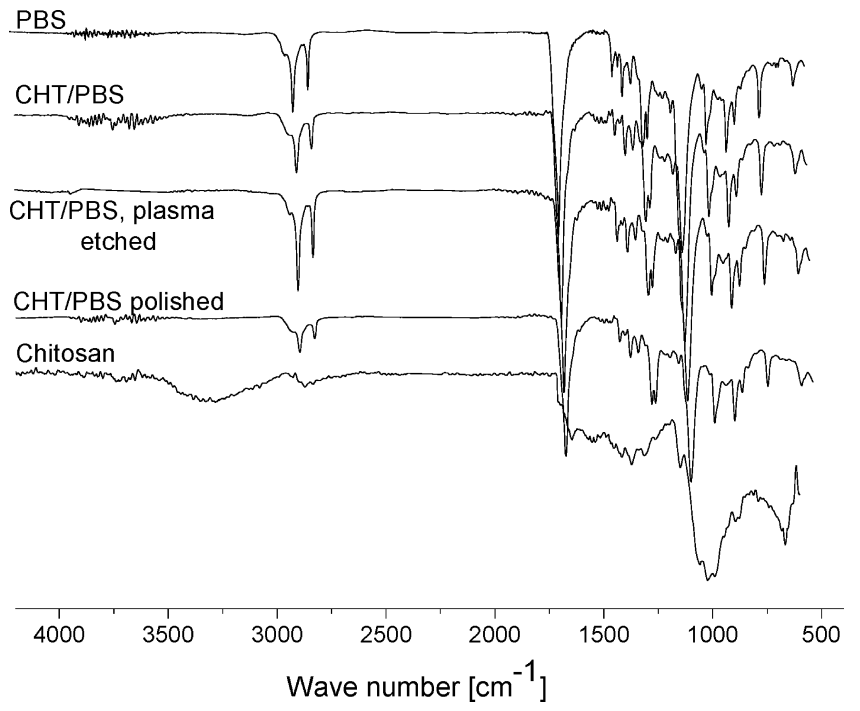

Figure 4. FTIR-ATR spectra of PBS, chitosan, and of the CHT/PBS blend before and after etching.

Table 1. Characteristic Absorption Bands of the Components of the CHT/PBS Blend

\begin{tabular}{llc}
\hline \multirow{2}{*}{ functional group } & \multicolumn{2}{c}{ wave number $\left[\mathrm{cm}^{-1}\right]$} \\
\cline { 2 - 3 } & \multicolumn{1}{c}{ chitosan } & poly(butylene-succinate) \\
\hline $\mathrm{O}-\mathrm{H}$ and $\mathrm{N}-\mathrm{H}$ & $3480-3080$ \\
$\mathrm{CH} \mathrm{H}_{2}$ & $2960-2560$ & \\
$\mathrm{C}=\mathrm{O}$ & 1648 & 1713 \\
$\mathrm{NH}_{2}$ & 1396 & \\
$\mathrm{C}-\mathrm{O}$ (and $\mathrm{C}-\mathrm{N}$ for $\mathrm{CHT})$ & $1139-915$ & 1157 \\
$\mathrm{C}-\mathrm{H}$ & & $1147-1263$ \\
\hline
\end{tabular}

Table 2. Chemical Composition of Untreated and Treated Materials Determined by XPS

\begin{tabular}{lcccc}
\hline \multicolumn{1}{c}{ materials } & $\mathrm{C}_{1 \mathrm{~s}}(\%)$ & $\mathrm{N}_{1 \mathrm{~s}}(\%)$ & $\mathrm{O}_{1 \mathrm{~s}}(\%)$ & $\mathrm{C}: \mathrm{O}$ ratio \\
\hline PBS & 83.53 & 0.08 & 16.39 & $1: 0.20$ \\
CHT/PBS & 74.01 & 0.15 & 25.84 & $1: 0.35$ \\
CHT/PBS, plasma etched & 77.41 & 0.42 & 22.18 & $1: 0.29$ \\
CHT/PBS, polished & 69.98 & 2.24 & 27.78 & $1: 0.40$ \\
\hline
\end{tabular}

a maximum depth of $250 \AA$, ${ }^{19}$ was performed to complement the surface chemistry information. Data for treated and untreated samples are summarized in Table 2.

The survey spectrum of PBS showed the presence of oxygen and carbon as it was expected considering its chemical structure (Figure 1). The nitrogen containing groups $\left(-\mathrm{NH}_{2}\right.$ and $-\mathrm{NH}-$ ) distinguished chitosan from PBS and an additional peak, corresponding to nitrogen, would be expected to appear in the spectrum of the blend. However, only traces of nitrogen (Table 2) were detected for the CHT/PBS blend. This result confirmed the observed skin-core morphology in which the very top surface layer was composed mainly by PBS and the chitosan content (as identified by the presence of nitrogen) increased with the depth. The highest nitrogen content observed in the bulk of the sample further confirmed the expected results.

The morphological and chemical surface changes detected after etching were further characterized by contact angle measurements (Figure 5).

The aliphatic polyester PBS presented a quite hydrophobic character with a contact angle of $107^{\circ}$. Contrary to PBS, the chitosan structure is rich in polar groups $\left(-\mathrm{OH}\right.$ and $\left.-\mathrm{NH}_{2}\right)$ therefore, its presence in the blend resulted in a significant decrease of the water contact angle. This reduction was even more notorious (contact angle of $78^{\circ}$ ) after removing the very

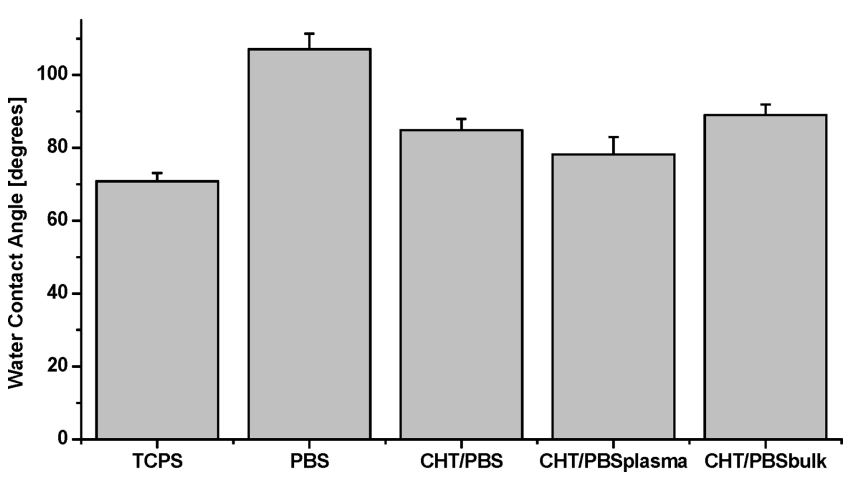

Figure 5. Water contact angles (mean \pm SD) for TCPS, PBS, untreated, and etched $\mathrm{CHT} / \mathrm{PBS}$ blend.

thin surface layer of the CHT/PBS samples by plasma etching. A more hydrophilic behavior was expected for the inner core of the material, due to an increase in chitosan content. However, the Cassie effect ${ }^{20}$ was observed for the polished samples and higher contact angle values were measured in this condition.

An earlier study ${ }^{21}$ reported that materials with high surface energy promote rapid cellular adhesion and spreading, whereas low surface energy do not favor such behavior. The surface energy of the studied materials was calculated using the Owens, Wendt, Rabel, and Kaeble (OWRK) method. ${ }^{15}$ The results are summarized in Table 3. The surface energy of the blend was significantly higher than that calculated for PBS. Moreover, the etching of the blend by plasma did not induce significant differences. However, the obtained value for the CHT/PBS is much lower $(10 \mathrm{mN} / \mathrm{m})$ than the TCPS surface energy. According to another study ${ }^{6}$ cell attachment can be correlated with the polar component of the surface energy, as denoted by the OWRK method, but not with the total surface energy. As can be seen from Table 3, this component increases with the exposure of the chitosan; similar values were obtained for the plasma-etched material and for TCPS. The higher content of chitosan in the bulk of the material was expected to further increase the polar component. However, the roughness of the surface after polishing did not allow a correct determination of the surface energy and its components.

Understanding how the characterized materials communicate with proteins and cells is of utmost importance to predict and ultimately modulate their behavior in the in vivo environment. During contact of surfaces with biological fluids, protein adsorption occurs almost instantaneously and it is this layer of biomolecules that mediates the key bio/nonbio interactions. Protein adsorption plays, therefore, a fundamental role in dictating the cellular response elicited by biomedical systems implanted in the human body. Generally, all studied surfaces showed preferential adsorption of human serum albumin (Figure 6). The interaction of proteins with surfaces is a complex process that depends on several parameters including the size and the conformation of the proteins. HFN and HSA have very different properties. HSA is a nonadhesive protein with molecular weight of $66.4 \mathrm{kDa},{ }^{22}$ while HFN is a cell-adhesive biomolecule with a molecular weight of $440 \mathrm{kDa}^{23}$ At the conditions of the assay (static, $2 \mathrm{~h}$ ), the smaller HSA reached the material surface faster than HFN. Furthermore, HSA being a nonselective biomacromolecule, tends to adsorb to different surfaces regardless the electrostatic interactions. $^{24}$

The lowest amount of the adsorbed proteins was registered for PBS, regardless the studied protein. This amount increased for the blend of PBS with chitosan. However, the measured difference was not statistically significant neither for CHT/PBS 
Table 3. Comparison of the Surface Energy and Respective Polar and Dispersive Components of the Studied Materials and TCPS

\begin{tabular}{lccr}
\multicolumn{1}{c}{ materials } & surface energy $(\mathrm{mN} / \mathrm{m})$ & polar component $(\mathrm{mN} / \mathrm{m})$ & dispersive component $(\mathrm{mN} / \mathrm{m})$ \\
\hline PBS & $22.68 \pm 0.59$ & $0.00 \pm 0.00$ & $22.68 \pm 0.59$ \\
CHT/PBS & $28.96 \pm 1.11$ & $3.77 \pm 0.48$ & $25.19 \pm 1.00$ \\
CHT/PBS, plasma etched & $26.61 \pm 0.86$ & $9.27 \pm 0.76$ & $17.34 \pm 0.40$ \\
CHT/PBS, polished $^{a}$ & $22.81 \pm 0.49$ & $3.78 \pm 0.42$ & $19.03 \pm 0.25$ \\
TCPS & $38.90 \pm 0.40$ & $8.57 \pm 0.40$ & $30.33 \pm 0.21$ \\
\hline
\end{tabular}

${ }^{a}$ Surface energy was calculated from the contact angle values, influenced by the Cassie effect.

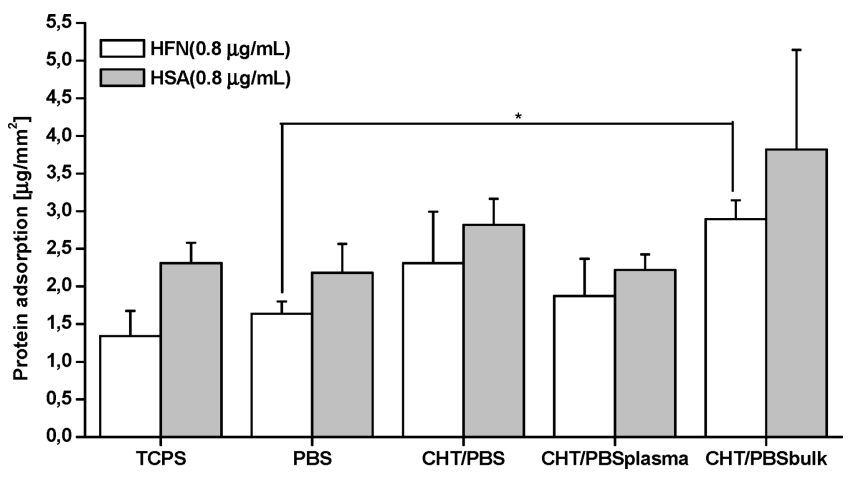

Figure 6. Protein adsorption onto TCPS, PBS, CHT/PBS, and etched surfaces after $2 \mathrm{~h}$ of incubation in HFN and HSA solutions. *Significantly different $(t$-test, $p<0.05)$.

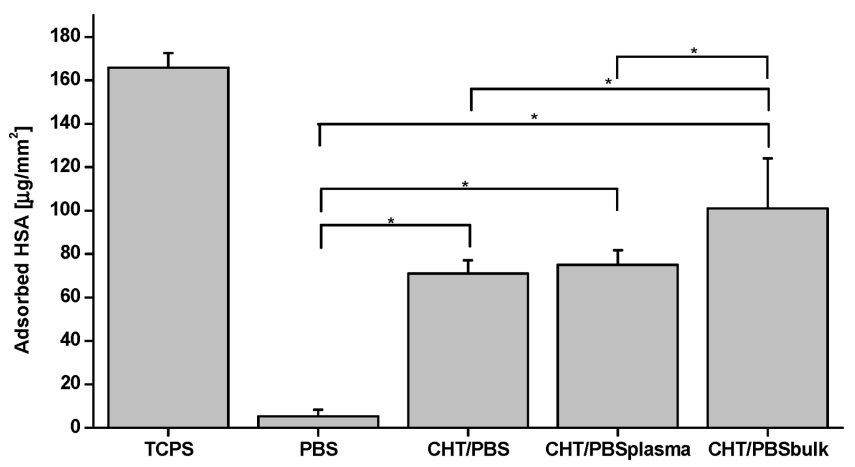

Figure 7. HSA adsorption from solution with concentration $70 \mu \mathrm{g} / \mathrm{mL}$ after $2 \mathrm{~h}$ of incubation. *Significantly different $(t$-test, $p<0.05)$.

nor for the blend etched with plasma. The highest level of protein adsorption was registered when the bulk of the material was exposed to the proteins $(p<0.05)$. These results were further confirmed by the experiments with $0.2 \%$ of the protein concentration in the human blood plasma $(0.8 \mu \mathrm{g} / \mathrm{mL}$ for $\mathrm{HFN}$ and $70 \mu \mathrm{g} / \mathrm{mL}$ for HSA). The quantities of HSA adsorbed from the solution with concentration $70 \mu \mathrm{g} / \mathrm{mL}$ were significantly higher (Figure 7) than those obtained with the initial concentration of $0.8 \mu \mathrm{g} / \mathrm{mL}$ (Figure 6). The only exception to this trend was observed for PBS. Those results indicated that at lower concentration, HSA was not sufficient to saturate the surface and adsorption points were probably still available.

The adsorption of HFN and HSA at concentrations similar to those of human blood showed highest adsorption of both proteins in the polished samples. According to already presented data, there are two main differences between this material and the other analyzed surfaces: highest chitosan content exposed at the surface and higher roughness, i.e. larger surface area. These two factors favor higher levels of protein adsorption. However, the statistically significant difference observed between the HSA adsorbed onto CHT/PBS (relatively flat surface) (Figure 7) and the one adsorbed onto PBS, makes clear that the presence of chitosan is more important than the increase in surface area.

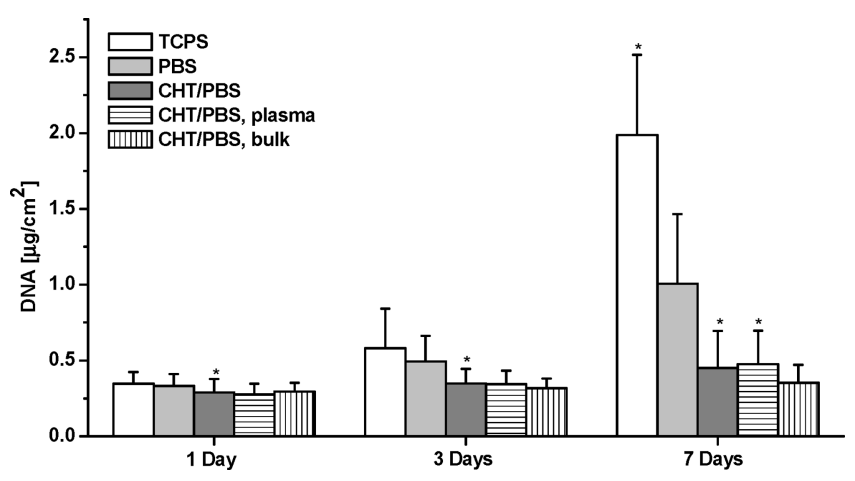

Figure 8. DNA concentrations corresponding to SaOs-2 cultured on PBS and CHT/PBS before and after etching for 1, 3, and 7 days. ${ }^{*}$ Not normal distribution (Shapiro-Wilk's test).

The in vitro biological performance of the studied surfaces was further assessed after culture with osteoblastic-like cells. SaOs-2 adhered to all of the tested materials after short culturing periods and proliferated at considerable rates in order to form an almost confluent layer of cells after 7 days of culture. Figure 8 shows the amount of DNA for the different time periods. The DNA amount increased with time of culture for all studied materials, which confirmed cell proliferation. However, the proliferation rate was different for the various surfaces under study. Although at the initial period of culture (1 day), the difference between the surfaces was not clear, after 7 days of culture a noteworthy increase of DNA concentration was observed for the cells cultured on PBS. It should be noted, that the surface properties dictate the very first events occurring when the material becomes in a contact with the biological environment thus, at latter time stages the in vitro performance of the material is also affected by other parameters imposed by the presence of cells at the surface.

The SEM analysis of the adhered cells after 1 day of culture, on the different surfaces (Figure 9), provided further information regarding the influence of the surface properties over cell morphology. After 1 day of culture, the majority of the cells were attached but not spread on the surface of PBS (Figure 9A). This behavior was completely overcome in the blend (Figure 9B) and SaOs-2, cultured on this surface, showed a higher degree of spreading with some extended lamellipodia over the substrate. This effect was even more notorious after etching the blend by plasma (Figure 9C) or by polishing (Figure 9D). Cells were more flattened and with more adhesion points for the etched surfaces. According to the protein adsorption results, those were the surfaces with highest adsorptive potential for the adhesive HFN, which interacts with the cell surface proteoglycans inducing the reorganization of the cytoskeleton and the assembly of focal adhesions. ${ }^{25}$

The influence of the surface properties over the osteoblastic activity of adhered cells intended to complement the analysis of the biological performance of the materials in study. ALP activity is widely used as biochemical marker for determining the levels of osteoblastic activity. ${ }^{18}$ The enzyme activity was 

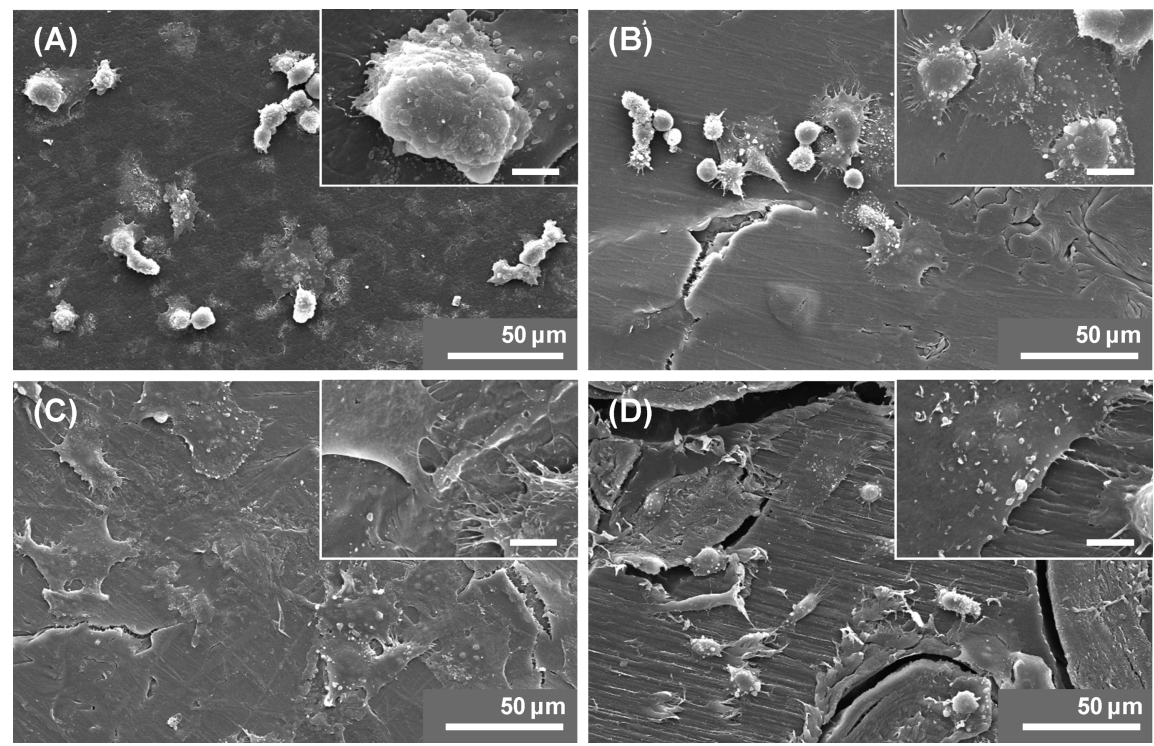

Figure 9. Scanning electron micrographs of osteoblast-like cells (SaOs-2), cultured for 1 day on PBS (A) and on CHT/PBS blend before (B) and after etching by plasma (C) and by polishing (D).

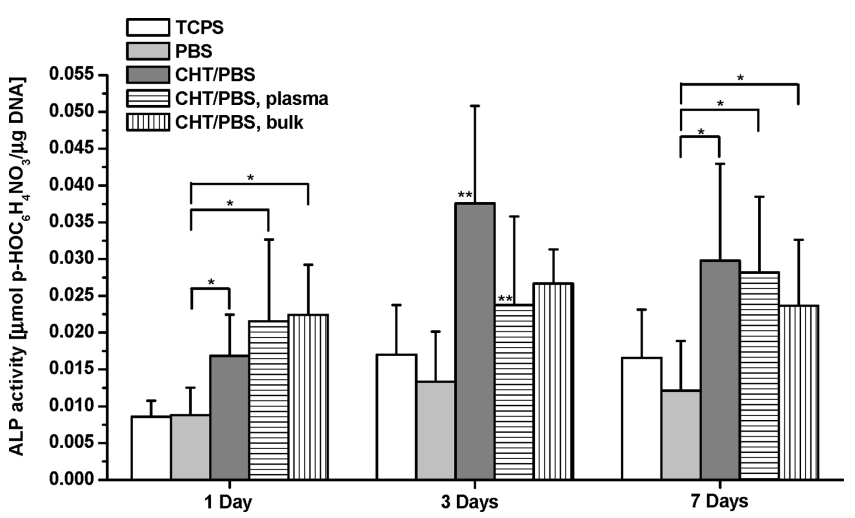

Figure 10. ALP activity/cell for osteoblast-like cells (SaOs-2) cultured in direct contact with PBS, CHT/PBS, CHT/PBS etched by plasma, and polished CHT/PBS. *Significantly different ( $t$-test, $p<0.05)$; ${ }^{*}$ Not normal distribution (Shapiro-Wilk's test).

shown to vary according to the surface properties of the studied materials (Figure 10). After 1 day of culture, the ALP activity was significantly higher for the chitosan-based blends and this tendency was kept for the longest time period, which presents an opposite tendency than that observed for cell proliferation. Other authors have reported similar results for a blend of poly(lactic acid-glycolic acid) (PLGA) with chitosan ${ }^{7}$ or for PLGA coated with chitosan. ${ }^{26}$ Higher ALP activity was measured in the presence of chitosan, while higher number of cells was registered for the PLGA only. Kim et al. ${ }^{27}$ also presented results showing the relation between protein adsorption levels and the improvement of ALP activity. These results are in accordance to those herein presented indicating that protein adsorption onto chitosan-based blends could be enhancing the expression of this biochemical marker.

\section{Conclusions}

An injection molded blend of chitosan with a synthetic polyester poly(butylene succinate) was herein studied. It was found that the blend presented a skin-core morphology with a surface layer of polyester and the chitosan component mostly dispersed in the core.

The removal of the surface layer by plasma etching or polishing exposed the chitosan component at the surface thus changing the biological performance of the material.
Regardless of the protein used, higher protein adsorption was registered for polished materials, which have higher chitosan content, in comparison to the other materials. Nonetheless, a preferential adsorption of HSA over HFN was registered for those samples.

The in vitro biological behavior of the CHT/PBS blend revealed the positive influence of the chitosan over osteoblastlike cells morphology and activity. Contrarily, higher proliferation rate was observed for the pure PBS.

This work confirmed that besides other aspects justifying the interest of combining synthetic and natural polymers, there is a distinctive advantage of using chitosan within this strategy to tailor the surface properties of a biomaterial thus improving its biological behavior.

Acknowledgment. The authors acknowledge the Foundation for Science and Technology (FCT), Portugal, for partial funding through FEDER and POCTI programmes and personal grant SFRH/BPD/8491/2002 (I. Pashkuleva). This work was carried out under the scope of the EU funded project HIPPOCRATES (NMP3-CT-2003-505758) and was partially supported by European Network of Excellence EXPERTISSUES (NMP3-CT2004-500283).

\section{References and Notes}

(1) Yi, H.; Wu, L.-Q.; Bentley, W. E.; Ghodssi, R.; Rubloff, G. W.; Culver, J. N.; Payne, G. F. Biomacromolecules 2005, 6 (6), 2881-2894.

(2) Sarasam, A. R.; Madhihally, S. Biomaterials 2005, 26 (27), 55005508.

(3) Sashiwa, H.; Aiba, S. Prog. Polym. Sci. 2004, 29 (9), 887-908.

(4) Rege, P. R.; Block, L. H. Carbohydr. Res. 1999, 321 (3-4), 235-245.

(5) Mori, T.; Okumura, M.; Matsuura, M.; Ueno, K.; Tokura, S.; Okamoto, Y.; Minami, S.; Fujinaga, T. Biomaterials 1997, 18 (13), 947-951.

(6) Lopez-Perez, P. M.; Marques, A. P.; da Silva, R. M. P.; Pashkuleva, I.; Reis, R. L. J. Mater. Chem. 2007, 17, 4064-4071.

(7) Jiang, T.; Abdel-Fattah, W.; Laurencin, C. T. Biomaterials 2006, 27 (28), 4894-4903.

(8) Correlo, V. M.; Boesel, L. F.; Bhattacharya, M.; Mano, J. F.; Neves, N. M.; Reis, R. L. Mater. Sci. Eng. 2005, 403 (1-2), 57-68.

(9) Correlo, V. M.; Pinho, E. D.; Pashkuleva, I.; Bhattacharya, M.; Neves, N. M.; Reis, R. L. Macromol. Biosci. 2007, 7 (3), 354-363.

(10) Ratner, B. D. Surface properties and surface characterization of materials. In Biomaterials Science: An Introduction to Materials in Medicine; Ratner, B. D., Hoffman, A. S., Schoen, F. J., Lemons, J. E., Eds.; Academic Press: New York, 2004. 
(11) Castner, D. G.; Ratner, B. D. Surf. Sci. 2002, 500 (1), 28-60.

(12) Zhao, B. H.; Lee, I. S.; Han, I. H.; Park, J. C.; Chung, S. M. Curr. Appl. Phys. 2007, 7S1, e6-e10.

(13) Anselme, K.; Bigerelle, M.; Noel, B.; Dufresne, E.; Judas, D.; Iost, A.; Hardouin, P. J. Biomed. Mater. Res. 2000, 49 (2), 155-166.

(14) Chatterjee, S.; Chatterjee, B. P.; Das, A. R.; Guha, A. K. J. Colloid Interface Sci. 2005, 208 (1), 30-35.

(15) Owens, D. K.; Wendt, R. C. J. Appl. Polym. Sci. 1969, 13 (8), 17411747.

(16) Kaelble, D. H. J. Adhes. 1970, 2, 66.

(17) Jenney, C. R.; Anderson, J. M. J. Biomed. Mater. Res. 1999, 49 (4), 435-447.

(18) Lian, J. B.; Stein, G. S. Crit. Rev. Oral Biol. Med. 1992, 3, 269-305

(19) Briggs, D. Surface Analysis of Polymers by XPS and Static SIMS; Cambridge University Press: New York, 1998.

(20) Cassie, A. B. D.; Baxter, S. Trans. Faraday Soc. 1944, 40, 546-551.
(21) Anselme, K. Biomaterials 2000, 21, 667-681.

(22) Curry, S.; Brick, P.; Franks, N. P. Biochim. Biophys. Acta 1999, 1441, 131-140.

(23) MacDonald, D. E.; Markovic, B.; Boskey, A. L.; Somasundaran, P. Colloids Surf., B 1998, 11 (3), 131-139.

(24) Nakanishi, K.; Sakiyama, T.; Imamura, K. J. Biosci. Bioeng. 2001, 91 (3), 233-244.

(25) Lyon, M.; Rushton, G.; Askari, J. A.; Humphries, M. J.; Gallagher, J. T. J. Biol. Chem. 2000, 275 (7), 4599-4606.

(26) Wu, Y.-C.; Shaw, S.-Y.; Linc, H.-R.; Leed, T.-M.; Yang, C.-Y. Biomaterials 2006, 27, 896-904.

(27) Zhao, G.; Schwartz, Z.; Wieland, M.; Rupp, F.; Geis-Gerstorfer, J.; Cochran, D. L.; Boyan, B. D. J. Biomed. Mater. Res., Part A 2004, $74 A$ (4), 49-58.

BM701268S 